\title{
Effect of a single leading-edge protuberance on NACA 634- 021 airfoil performance
}

\author{
Chang Cai ${ }^{1}$, Zhigang Zuo ${ }^{1}$, Shuhong Liu${ }^{1 *}$, Yulin Wu ${ }^{1,}$
}

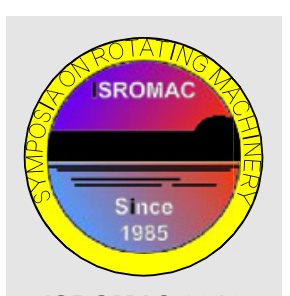

ISROMAC 2016

International

Symposium on Transport

Phenomena and Dynamics of

Rotating Machinery

Hawaii, Honolulu

April 10-15, 2016

\begin{abstract}
Leading-edge protuberances on airfoils or wings have been considered as a viable passive control method for flow separation. In this paper, the aerodynamic performance of a modified airfoil with a single leading-edge protuberances was investigated and compared with the baseline NACA 634-021. Spalart-Allmaras turbulence model was applied for numerical simulation and smoke flow was used for experimental visualization. Compared to the sharp decline of baseline lift coefficient, the stall angle of the modified foil is advanced and the decline of lift coefficient becomes mild. The post-stall performance of the modified airfoil is also improved. The attached flows along the peak of the protuberance help improving the total performance of the airfoil. Asymmetrical flows along the spanwise direction are observed on the modified airfoil, which may be responsible for the performance decline at pre-stall attack angles. The formation mechanism and suppression method of the symmetry breaking phenomenon should be investigated more deeply in the future to guide the practical application of the passive control method.
\end{abstract}

\footnotetext{
Keywords

Airfoil - Separation control — Leading-edge protuberance

${ }^{1}$ State Key Laboratory of Hydroscience and Engineering, Department of Thermal Engineering, Tsinghua University, Beijing, China
${ }^{*}$ Corresponding author: liushuhong@mail.tsinghua.edu.cn
}

\section{INTRODUCTION}

Stall occurs with a reduction in the lift coefficient generated by a foil when the critical angle of attacked is exceeded, accompanied with large region of flow separations. It would decrease the efficiency and performance of aircrafts, fluid machineries, or even lead to damage of the units. Therefore, separation control is very important and necessary.

Separation control can be achieved with active and passive methods. As active methods would always lead in complicated and expensive control system, the latter is considered to be more practical. In recent years, leadingedge protuberances, as a new passive control method, have drawn extensive attentions [1-4]. The idea came up from humpback whales, which are extremely maneuverable in spite of their large and rigid body. It has been hypothesized that the protuberance structures on the leading edge of their flippers are used for separation control, thus responsible for their high maneuverability [5].

The hydrodynamic performance of humpback whale flipper models was tested through experiment by Miklosovic et al. [6]. An increase in the maximum lift and an increase in the stall angle were found with protuberances. The effects of protuberances on two-dimensional airfoils, including NACA 634-021, 0021 and 65-021, have been explored by previous researchers [7-11]. Although the post-stall performance is also improved, the pre-stall performance suffered a drop to various degrees, making the current modification unpractical.

Custodio et al. [10] performed tuft and dye visualizations, finding that the spanwise flow between protuberances caused interferences between neighboring protuberances and generated a bi-periodic flow pattern, i.e., the near-wall flow of every two protuberances form a periodicity. A pair of counter-rotating vortices stemming from the leading edge was considered to help keeping the flow attached to the suction surface, similar to the effect of a delta wing. The bi-periodic condition was verified through numerical simulation performed by Cai et al., and different modes of spanwise periodicity were discovered [12]. However, the formation mechanism of spanwise periodicity is still unclear, which needs further investigation.

Most of the leading-edge protuberances were designed as continuous uniform distribution [7-12], and the flow patterns resulted from joint effects. In order to investigate the formation mechanism of spanwise periodicity, it is very necessary to examine the effect of a single protuberance, and further discuss the interferences of vortex structures between different protuberances. As a preliminary research, the effect of a single extended leading-edge protuberance on airfoil performance was investigated through numerical and experimental method in this paper.

\section{METHOD}

NACA $63_{4}-021$ airfoil, which is similar to the profile of the cross section of humpback whale flipper [1], is chosen as the baseline airfoil in this study (see Figure 1). The chord length $c=100 \mathrm{~mm}$ and the span $s=350 \mathrm{~mm}$. For the modified airfoil, an extended protuberance with sinusoidal leadingedge profile is located at the center of the foil span. The protuberance is formed by three characteristic foil profiles sweeping along the single-periodic sinusoidal leading edge, as shown in Figure 2. The wavelength of the protuberance 
$\lambda=25 \% c$ and the amplitudes $A=10 \% c$.

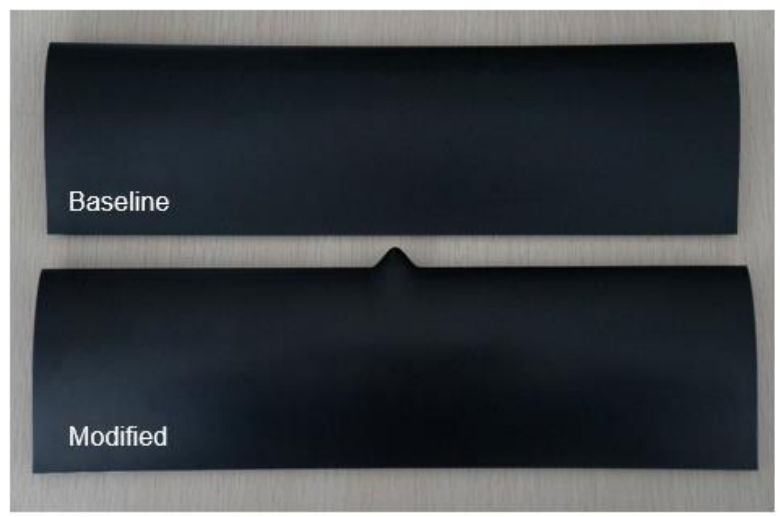

Figure 1. Experiment models

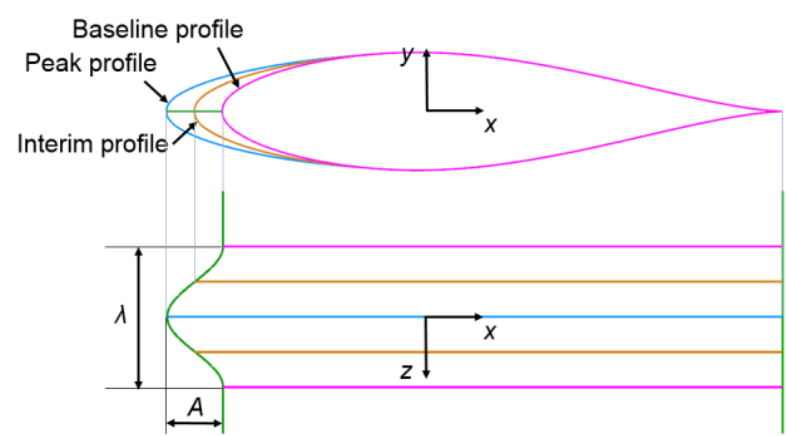

Figure 2. Detail of the leading-edge protuberance

\subsection{Numerical Method}

Hexahedral structured grids were built through O-type topological structure. The circumferential mesh around the leading and trailing edge as well as the spanwise mesh around the mid-section of Model $B$ is refined, as shown in Figure 3. The value of $y^{+}$around the foil is below 1. In order to reduce the disturbance of the boundary, the inlet, upper and lower wall is set $15 c$ away from the foil, while the outlet is $25 c$ away to keep the wake flow fully developed. The flanks of the computational domain were set as symmetry boundary condition. A mesh independence test was performed and shown in Figure 4. The total mesh number for the baseline and modified airfoil were respectively chosen as 3.7 and 6.1 million.

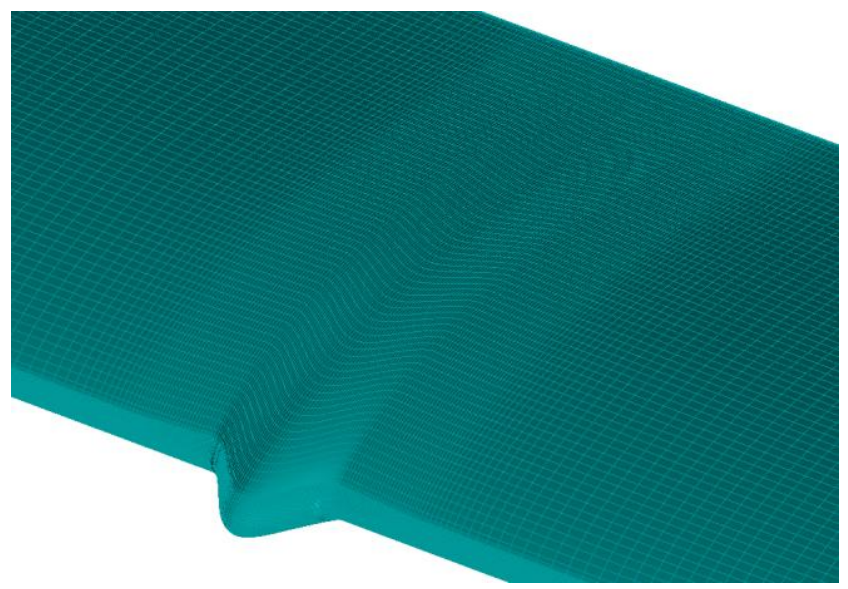

Figure 3. Surface mesh distribution

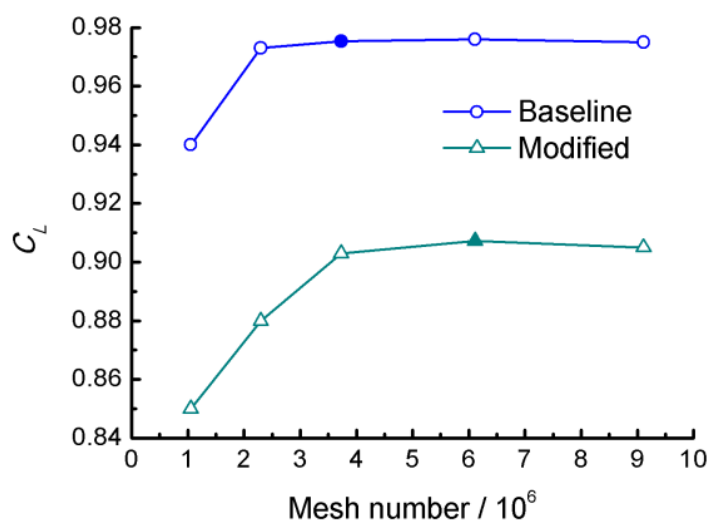

Figure 4. Mesh independence at $15^{\circ}$

The computations were carried out in FLUENT software solving Reynolds-Averaged Navier-Stokes (RANS) governing equations. The Spalart-Allmaras model, which has shown with pleasant results on wall-bounded flows in aero-space applications and relevant researches [12, 13], was applied for the turbulence modeling. The Reynolds number was $\mathrm{Re}=1.0 \times 10^{5}$.

\subsection{Experimental method}

Smoke flow method was adopted to view the situation of flow passing at different spanwise positions of the airfoil. Flow was seeded by smoke generated from vegetable oil and was illuminated by a NEW WAVE standard pulsed laser sources at a wavelength of $532 \mathrm{~nm}$ and a maximum energy output of 400 $\mathrm{mJ}$. The smoke flow images were taken by a Charge-Coupled Device camera (Nikon type 13, $1024 \times 1024$ pixels). A LaVision processor was used to synchronize image collection and illumination. Each image covered an area of $250 \mathrm{~mm} \times$ $250 \mathrm{~mm}$ for measurements.

Four $x-y$ sections were chosen for flow visualization, including the mid-section of both airfoils and the trough position at the protuberance, shown in Figure 5. The shooting frequency was $4 \mathrm{fps} .150$ images were taken for each section.

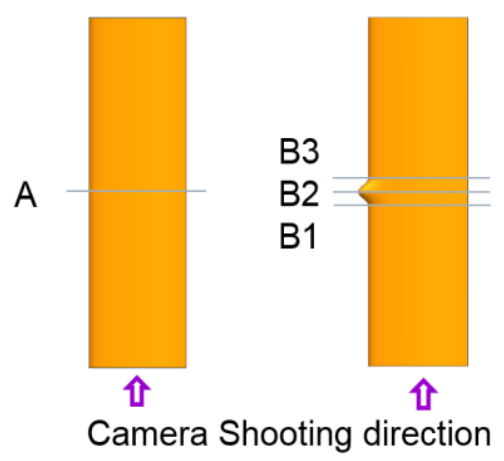

Figure 5. Test sections for smoke flow visualization

\section{RESULTS AND DISCUSSION}

\subsection{Performance comparison}

The calculated lift and drag coefficient alteration with different attack angles are compared with experiment data from Ref. [7, 8] in Figure 6 and 7. For the baseline NACA 634-021 airfoil, 
good agreement is demonstrated while the Reynold number is a bit different. Overall, the simulation results fit better with the experiment at higher Reynolds number $\mathrm{Re}=1.8 \times 10^{5}[8]$. The reason may be ascribed to the fully turbulence model applied in current simulation, which may be flawed for laminar or transition flow characteristics at lower Reynolds number.

For the Baseline foil, the lift coefficient increases linearly from $0^{\circ}$ to $12^{\circ}$, and the growth slows down from $12^{\circ}$ to $18^{\circ}$. Stall occurs at around $18^{\circ}$, with a great loss in lift and large increases in drag. For the modified airfoil, the attack angle with maximum lift was advanced to about $15^{\circ}$, while the decline of lift turns out much gentler. The deep-stall lift is very close to the baseline foil. The drag coefficients of the modified foil are a bit larger than the baseline between $12^{\circ} \sim 18^{\circ}$, while at smaller and larger attack angles shows few differences.

It should be noticed that the local chord length with the protubenrance is increased, thus the total planform area of the modified airfoil is $0.36 \%$ larger than the baseline. However, Table 1 shows the relative change ratio of lift and drag coefficient of the modified airfoil, which is much more remarkable. With the protuberance, the performance at pre-stall attack angles is deteriorated, with approximately $10 \%$ lower lift and $50 \%$ to $80 \%$ higher drag.

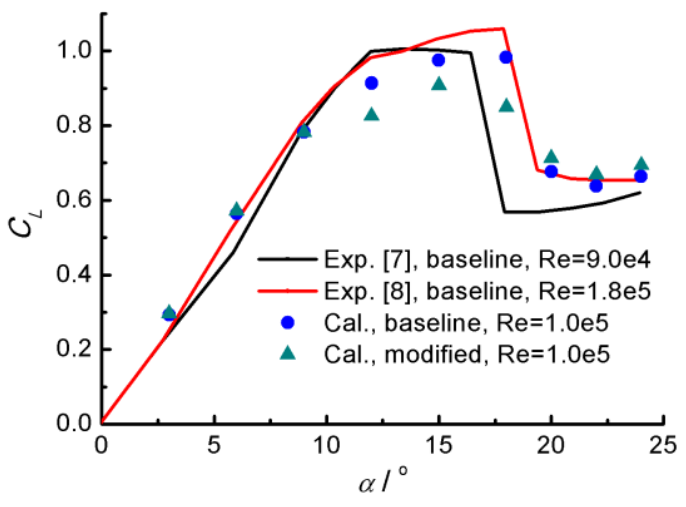

Figure 6. Lift performance (Experiment: [])

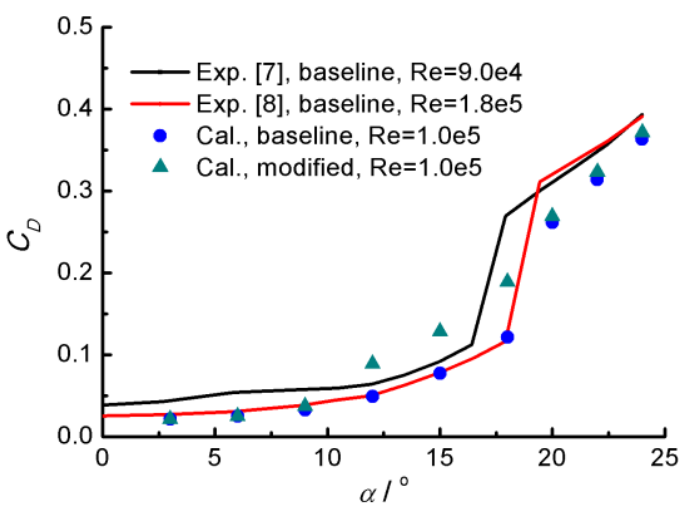

Figure 7. Drag performance (Experiment: [])
Table 1. Relative change ratio of lift and drag coefficient of the modified airfoil

\begin{tabular}{ccc}
\hline$\alpha /^{\circ}$ & $\Delta C_{L} / \%$ & $\Delta C_{D} / \%$ \\
\hline 3 & 1.4 & 4.7 \\
6 & 1.3 & 12.0 \\
9 & -0.1 & 14.1 \\
12 & -9.6 & 80.0 \\
15 & -7.0 & 65.6 \\
18 & -13.5 & 56.0 \\
20 & 5.3 & 2.9 \\
22 & 4.9 & 2.9 \\
24 & 4.5 & 2.3 \\
\hline
\end{tabular}

\subsection{Flow characteristics}

Figure 8 shows the limiting streamline on the suction surface as well as the recirculation regions shown by isosurfaces of longitudinal velocity $V_{x}=0$ from $9^{\circ}$ to $24^{\circ}$. At $9^{\circ}$, the flow keep attached along the peak section of the protuberance, while larger recirculation occurs at the flanks nearly symmetrically. The influence range of the protuberance is very limited, since similar recirculation position and area can be observed on most region of the suction surface. Correspondingly, the force performance of the modified airfoil is very close to the baseline at $9^{\circ}$.

At $15^{\circ}$, the separation line of the baseline airfoil moves towards the leading edge and the recirculation region enlarges slightly, and the flow remain two-dimensional. However, the flow through the modified airfoil turns out to be asymmetric. A number of limiting streamlines tilt towards the right side of the protuberance and keep towards the flow direction until reaching the trailing edge. As for the left side of the protuberance, a large region of recirculation occurs with leading-edge separation. Encountering early stall locally, the large separation would lead to a decline of lift and increase of drag. The influence of the protuberance extends to the whole span, even changing the separation condition at the span ends.

At $20^{\circ}$ where stall occurs, the separation line moves further forward to the leading edge with slightly threedimensional effect. For the modified airfoil, an attached flow also locates on the right side of the protuberance before the mid-chord. The other region on the suction surface demonstrate a similar condition as the baseline airfoil.

At $24^{\circ}$, the whole suction surface of the baseline airfoil is in separation and return to two-dimensional condition. As to the modified airfoil, an attached flow originated from the peak section of the protuberance tilts towards left, leading to a smaller recirculation region behind. The attached flow at $20^{\circ}$ and $24^{\circ}$ will improve the local performance of the airfoil, therefore the total lift coefficient increases slightly. 


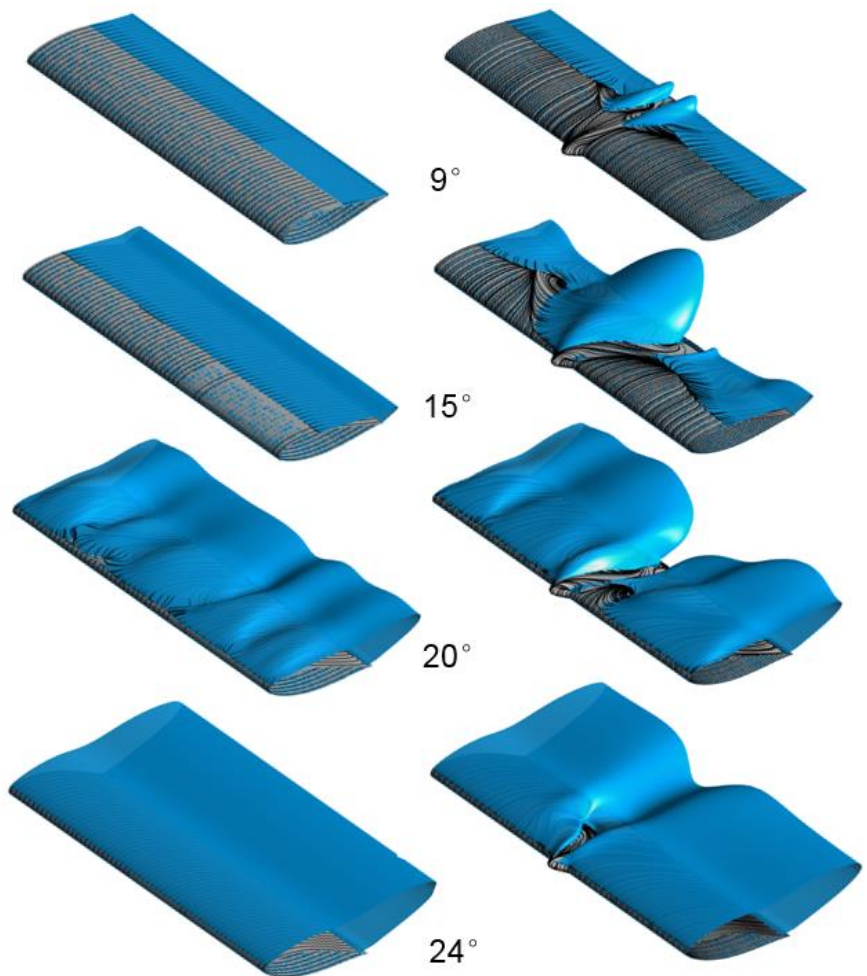

Figure 8. Limiting streamlines and recirculation regions shown by isosurface of zero longitudinal velocity (Left: baseline; right: modified)

Flow asymmetry passing the leading-edge protuberance can be observed at high angles of attack in Figure 9. In order to examine the existence of asymmetry, smoke flow visualization at different spanwise positions was performed, see Figure 5. Figure 8 shows the instantaneous flow at Sec. A at $15^{\circ}$ and $24^{\circ}$. Considering the nonumiformity of smoke and the low shooting frequency (4fps) which failed to capture the dynamic evolution of flow structures, the 150 images for each section were averaged in the post-processing. The processing method may eliminate some flow phenomenon such as separation bubble or reattachment. However, it would be capable to show the separation position and the region of separation vortex intuitively. The averaged results are shown in Figure 10 and 11 and compared with the streamlines from numerical simulation. The foil profile at each section are highlighted and the separation position are indicated generally. It should be noticed that at Sec. B3, the flow past the leading edge would be partially shielded by the protuberance due to the shooting perspective.
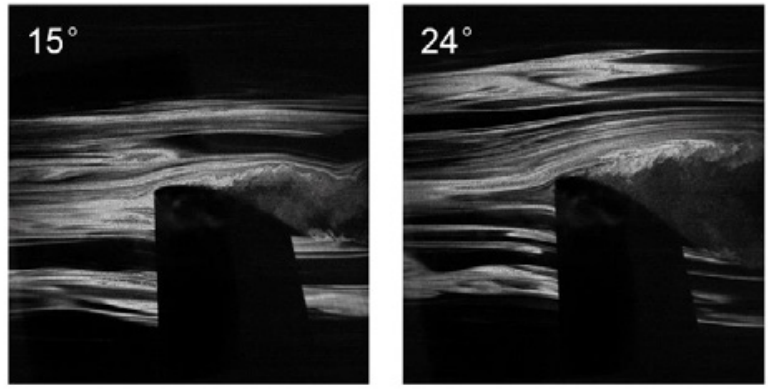

Figure 9. Instantaneous image of smoke flow at Sec. A
Figure 9 shows the flows at different sections at $15^{\circ}$. For Sec. A, the visualization results are slightly different between the experiment and simulation. The simulation separation point is relatively more downstream and the separation region is smaller. However, for Sec. B1 B3 of the modified airfoil, the experiment and simulation results share high similarity. The separation point at Sec. B1 is very close to Sec. A, while the range of separation vortex is larger. At Sec. B3, the separation point locates at around the leading edge, with large separation vortex covering the whole suction surface. The flow situation at Sec. 2 with maximum foil profile is between B1 and B3. It should be noticed that the spanwise positions of $B 1$ and B3 are symmetric with respect to the mid-section B2, while remarkable difference exists between the flow field. The larger separation around Sec. B2 and B3 would lead to the lift decline and drag increase at pre-stall angles.
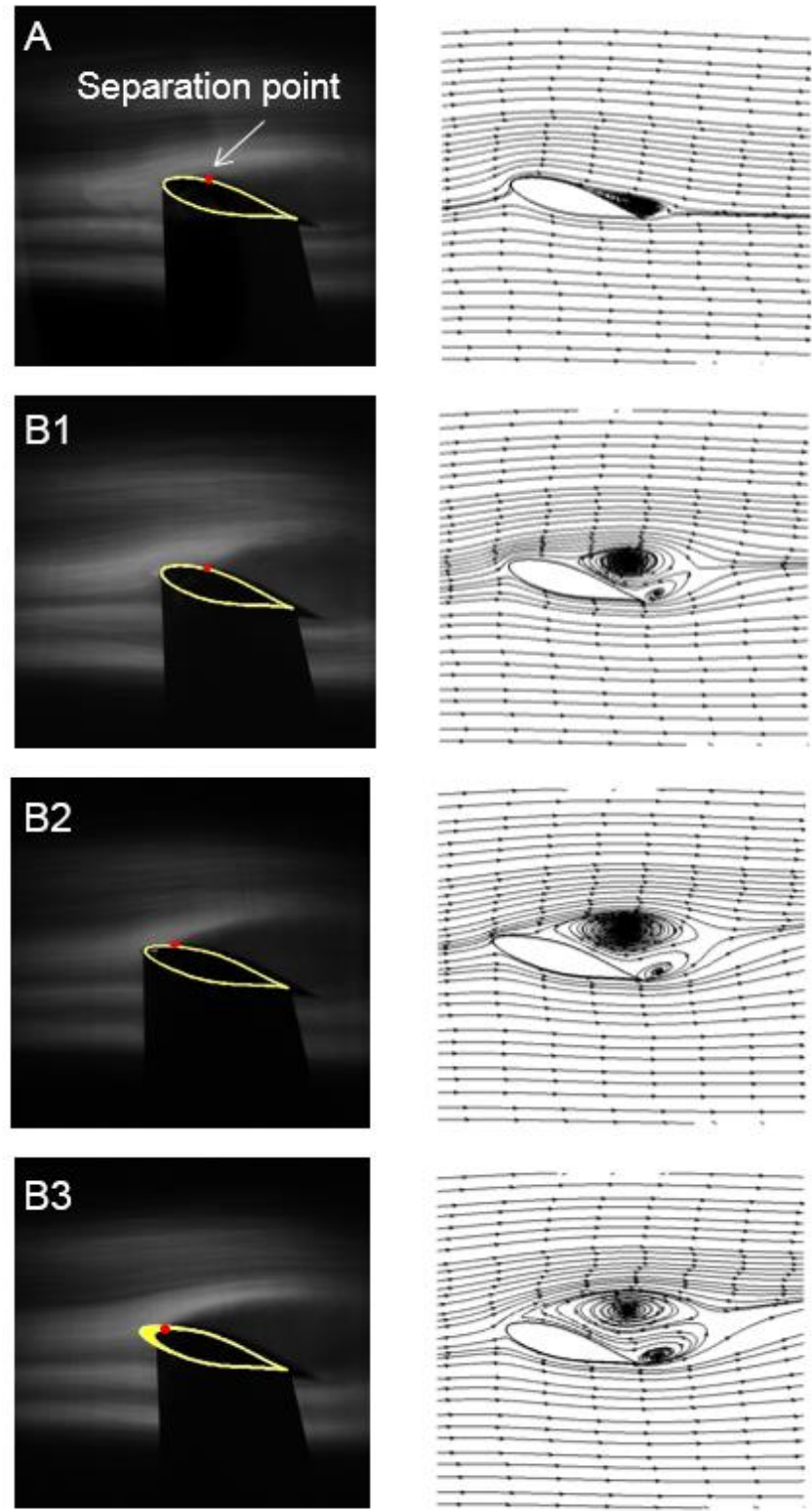

Figure 10. Averaged image of smoke flow (left) and 2D streamlines gained by numerical simulation (right) at $15^{\circ}$ 
Effect of a Single Leading-edge Protuberance on NACA 63 ${ }_{4}-021$ Airfoil Performance - 5

Figure 11 shows the flows at $24^{\circ}$. The baseline airfoil suffers from leading edge separation, which is shown by experiment and simutation consistently. As for the modified airfoil, the flow at Sec. B1 is very similar to that at Sec. A. The flow keep attached along $1 / 4$ chord at Sec. B2 and along half chord at Sec. B3. The difference between Sec. B1 and $B 3$ verifies the asymmetry phenomenon again. The attached flow around Sec. B2 and B3 may be responsible for the lift increase at post-stall angles.
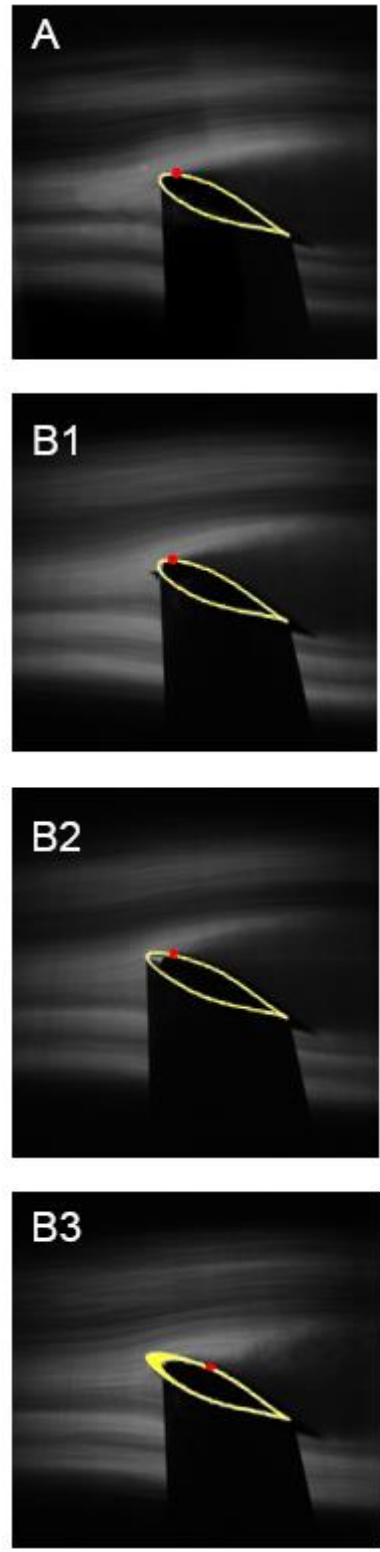
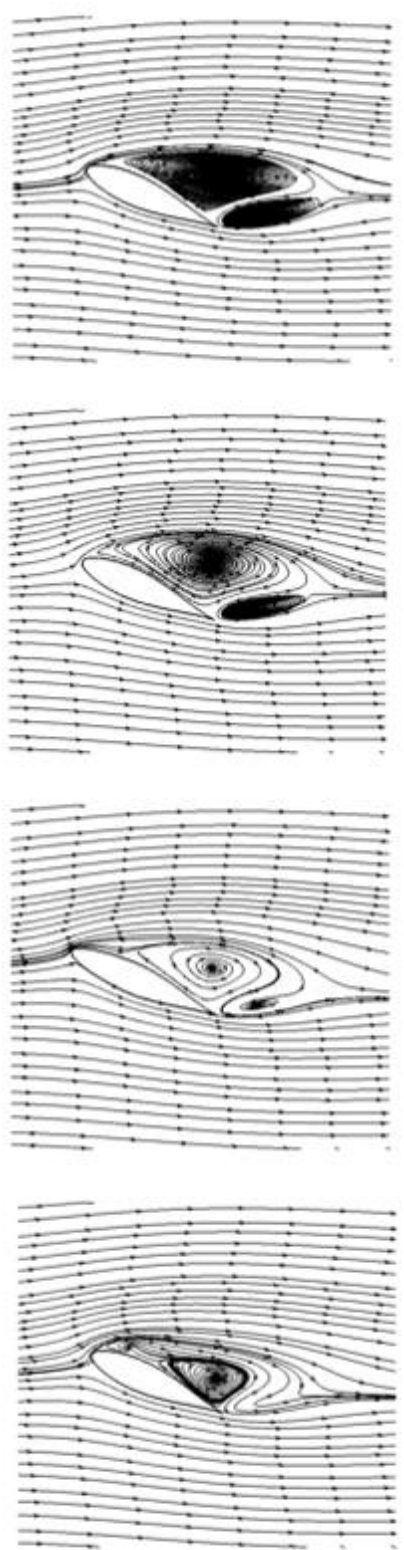

Figure 11. Averaged image of smoke flow (left) and $2 \mathrm{D}$ streamlines gained by numerical simulation (right) at $24^{\circ}$

Figure 12 shows the streamwise vorticity, which is defined as

$$
\omega_{x}=\frac{\partial V_{z}}{\partial y}-\frac{\partial V_{y}}{\partial z},
$$

at different streamwise positions at $15^{\circ}$. A couple of sheet streamwise vorticity is formed above the leading-edge protuberance symmetrically at initial stage. With the flow develop downstream, the negative vorticity on the right raises from the surface and furtherly evolves into complicated vortex structure, while the positive vorticity keeps as the shape of sheet. The asymmetrical development of the streamwise vorticity and the complicated interferences with separation vortices may give rise to the special flow pattern and performance of the moidified airfoil.
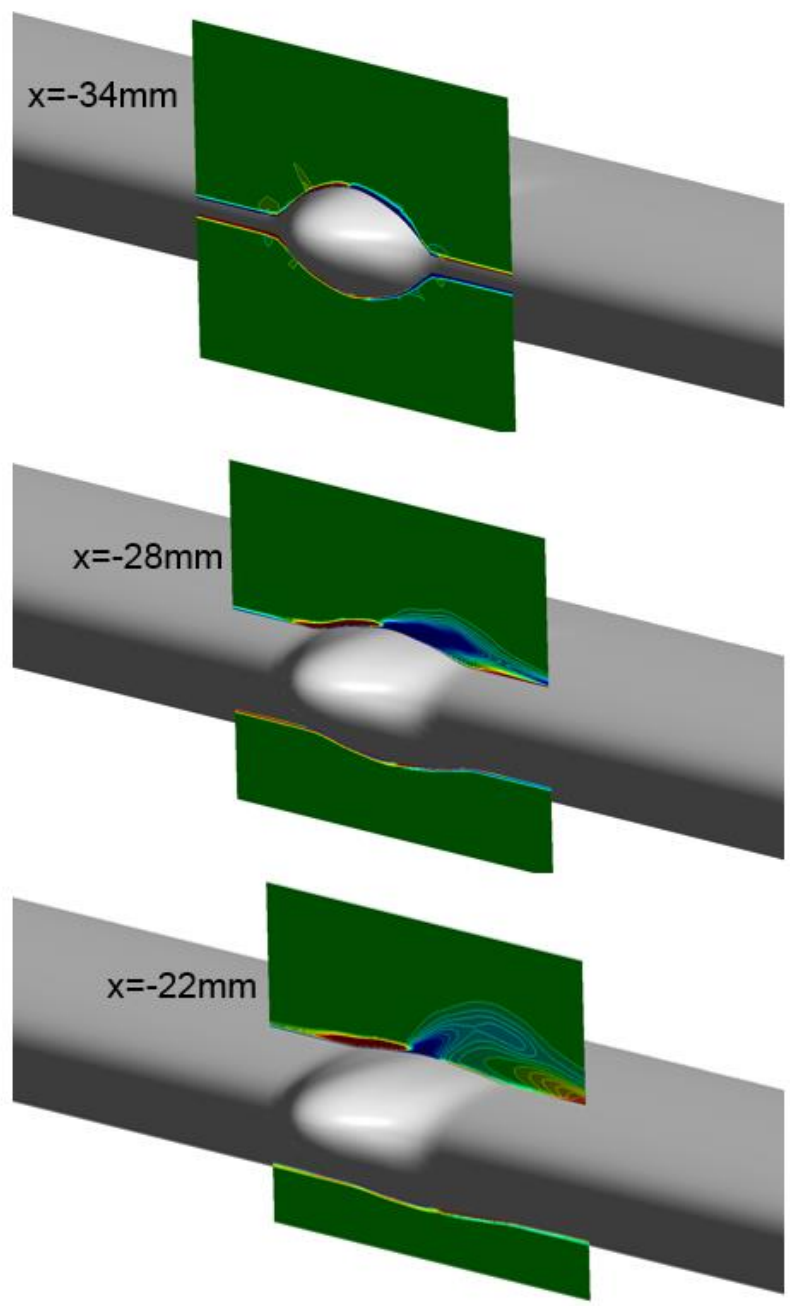

Figure 12. Streamwise vorticity development at $15^{\circ}$

\subsection{Discussion}

In order to check whether the asymmetry phenomenon is caused by numerical method such as turbulence model, SST $k-\omega$ model was also applied in this research. Figure 13 shows the limiting streamlines and recirculation regions of the modified airfoil at $15^{\circ}$. Although the result is a bit different from that in Figure 8, strong asymmetry can also be observed. Therefore, the influence of turbulence model is excluded and the aymmetry phenomenon may probably be physical. More precise method such as DES or LES would be carried out to check the detailed vortex structures and their development. 
Effect of a Single Leading-edge Protuberance on NACA 63 ${ }_{4}-021$ Airfoil Performance - 6

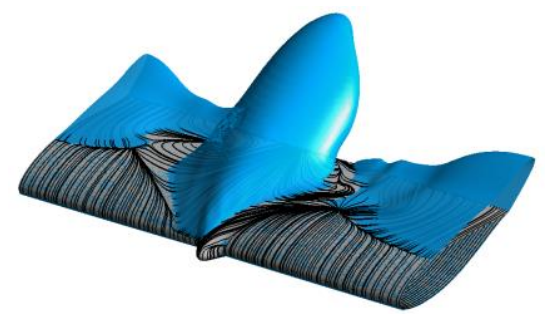

Figure 8. Limiting streamlines and recirculation regions shown by isosurface of zero longitudinal velocity (SST $k-\omega$, modified airfoil, $15^{\circ}$ )

The formation and development of streamwise vorticity in Figure 12 may be regarded as similar to the leading-edge vortices on delta wings. Due to the special shape of protuberance expecially the round and thick leading edge compared to traditional delta wings, the streamwise vortices in the primary stage turn out to be sheet shaped. It is important to notice that symmetry breaking of leading-edge vortices is a very common phenonmenon on delta wings [14] which may explain the asymmetry condition past the protuberance discovered in this research.

It can be speculated that if the leading-edge protuberances were ranged as continuous uniform distribution, the asymmetrical vortex structure induced by different protuberances would have complicated interferences with each other, thus forming different flow conditions such as bi-periodicity. Stability theory may be capable to explain the symmetry breaking of streamwise vortices and formation of bi-periodic structures, which will be the key of our future job.

\section{CONCLUDING REMARKS}

In this paper, the effect of an single leading-edge protuberance on the performance of NACA 634-021 was investigated. At post-stall angles of attack, the attached flow along the peak of the protuberance would reduce the local separation around, thus improving the total performance of the airfoil slightly. However, lift loss and drag increase at post-stall attack angles is accomapanied. Asymmetrical flow condition is observed through both numerical simulation and smoke flow visualization. The severe spanwise flow and advanced stall region may be responsible for the performance decline. In order to minimize the decline of post-stall performance, and furtherly make the passive control method more practical, the formation mechanism and suppression method of the symmetry breaking phenomenon should be investigated more deeply in the future.

\section{REFERENCES}

[1] Fish F E, Battle J M. Hydrodynamic design of the humpback whale flipper[J]. Journal of Morphology, 1995, 225(1): 51-60.

[2] Fish F E, Lauder G V. Passive and active flow control by swimming fishes and mammals[J]. Annu. Rev. Fluid Mech., 2006, 38: 193-224.

[3] Fish F E, Howle L E, Murray M M. Hydrodynamic flow control in marine mammals[J]. Integrative and Comparative Biology, 2008, 48(6): 788-800.
[4] Fish F E, Weber P W, Murray M M, et al. The Tubercles on Humpback Whales' Flippers: Application of BioInspired Technology[J]. Integrative and comparative biology, 2011, 51(1): 203-213.

[5] Fish F E. Performance constraints on the maneuverability of flexible and rigid biological systems[C]//Proceedings of the Eleventh International Symposium on Unmanned Untethered Submersible Technology. 1999: 394406.

[6] Miklosovic D S, Murray M M, Howle L E, et al. Leading-edge tubercles delay stall on humpback whale (Megaptera novaeangliae) flippers[J]. Physics of Fluids (1994-present), 2004, 16(5): L39L42.

[7] Johari $\mathrm{H}$, Henoch C W, Custodio D, et al. Effects of leading-edge protuberances on airfoil performance[J]. AIAA journal, 2007, 45(11): 26342642.

[8] Johari H. Applications of hydrofoils with leading edge protuberances[R]. CALIFORNIA STATE UNIV NORTHRIDGE DEPT OF MECHANICAL ENGINEERING, 2012.

[9] Hansen K L, Kelso R M, Dally B B. The effect of leading edge tubercle geometry on the performance of different airfoils[J]. World, 2009.

[10] Custodio D. The effect of humpback whale-like leading edge protuberances on hydrofoil performance[D]. Worcester Polytechnic Institute, 2007.

[11] Zhang M M, Wang G F, Xu J Z. Experimental study of flow separation control on a low-Re airfoil using leading-edge protuberance method[J]. Experiments in Fluids, 2014, 55(4): 1-13.

[12] Cai C, Zuo Z, Liu S, et al. Numerical investigations of hydrodynamic performance of hydrofoils with leading-edge protuberances[J]. Advances in Mechanical Engineering, 2015, 7(7).

[13] Dropkin A, Custodio D, Henoch C W, et al. Computation of Flow Field Around an Airfoil with Leading-Edge Protuberances[J]. Journal of Aircraft, 2012, 49(5): 1345-1355.

[14] Gursul I. Review of unsteady vortex flows over slender delta wings[J]. Journal of Aircraft, 2005, 42(2): 299-319. 